\title{
Nanoparticle coagulation and dispersion in a rotating curved pipe
}

Nanoparticulate flow in a curved pipe differs to that in a straight pipe, and is of scientific and technological interest. The length of flow path within a curved pipe is generally short, and the fluid experiences a centrifugal force which significantly changes the particle distribution both inside and downstream of the pipe. This phenomenon is more obvious when particle coagulation occurs. Prior to this study, nanoparticle migration in a rotating curved pipe with consideration of coagulation and dispersion has not been reported in detail. This study aimed to investigate the evolution of particle number, mass, polydispersity, diameter and geometric standard deviation in a rotating curved pipe, with consideration of coagulation and dispersion, at different Reynolds, Schmidt and F numbers using the Taylor-expansion moment method. Numerical results of axial velocity and secondary streamline are compared with previously reported results for validation. The following conclusions are drawn.

When the Coriolis and centrifugal forces are in the same direction, particles progressively concentrate near the outside edge of the pipe with time. The maximum particle number appears in the region where the axial velocity is the highest. The particle number increases rapidly at first and then approaches a stable value, which is different to the case of micro-sized particles. The distribution pattern of particle mass is similar to that of particle number, in that particle mass over the cross-section increases with time. In an initially monodisperse particle field, coagulation produces particles of various size and then increases in particle size. As coagulation proceeds, particle diameter, polydispersity and geometric standard deviation all increase. The incremental change in particle polydispersity is initially small because particles seldom collide, but later increases drastically. There exists a crescent region near the outer edge of the pipe where the particle diameter is large. Regions with large particle diameters correspond to those with high particle number and larger geometric standard deviation. The particle diameter and geometric standard deviation progressively increase from an initial value to differing values dependent on position.

When the Coriolis and centrifugal forces are in opposite directions and the Coriolis force is dominant, particles concentrate near the inside edge of the pipe. A maximum particle number and mass is again apparent in the region where the axial velocity is the highest. Particles in the high particle number region also have greater mass, diameter, polydispersity and geometric standard deviation. Thus, in a rotating curved pipe particle distribution depends on the balance of the Coriolis and centrifugal forces, i.e. pipe curvature and rotation speed. Particles will accumulate near the outside edge if the centrifugal force is dominant, and near the inside edge if the Coriolis force is dominant.

The Reynolds and Schmidt numbers have an effect on particle distribution when other parameters are constant. An increase in Reynolds number leads to an increase in particle number and mass, and a decrease in particle polydispersity, diameter and geometric standard deviation which is attributed to the high coagulation rate at low flow. A large Schmidt number corresponds to a small particle dispersion and therefore a small probability of particle coagulation. An increase in Schmidt number leads to an increase in particle number and mass, and a decrease in particle polydispersity, diameter and geometric standard deviation.

See the article: Lin J Z, Lin P F, Chen H J. Nanoparticle distribution in a rotating curved pipe considering coagulation and dispersion. Sci China Phys Mech Astron, 2011, 54: 1502-1513

Open Access This article is distributed under the terms of the Creative Commons Attribution License which permits any use, distribution, and reproduction in any medium, provided the original author(s) and source are credited. 